of liberal-humanist criticism. But having in the first book seen off in style his two leading players, he has wanted somehow to pull together the remaining cast into a separate production. The task was not really feasible.

Heims's manner of falling short, however, cheats the reader of a legitimate expectation. Rather than assurances that cybernetics still lives, the reader may prefer particulars of the accomplishments of its lifetime and an

\section{Telling differences}

\author{
Stuart Sutherland
}

Perceptual and Associative Learning. By Geoffrey Hall. Oxford University Press: 1991. Pp. 300. £30, $\$ 45$.

AsSOCIATIONISM, the doctrine that all mental events (and the ensuing behaviour) can be explained in terms of the association of ideas, was first systematically propounded by David Hume. At some level it must be correct: after all, the operation of the nervous system is governed by the connections between its cells. The real issues are what can be associated with what and how the associations are determined. Until recently, most workers in animal learning, such as Clarke Hull and B. F. Skinner, have assumed that the only associations made were between stimuli and responses ( $\mathrm{S}$ $\mathrm{R}$ theory) and that the strength of an association depended on the number of times reinforcements were given. Both assumptions have been discarded. Nowadays, all agree that connections between stimuli can be formed (S-S learning), and that, even if a reinforcer always follows a stimulus, no connection will be made if the reinforcer occurs as frequently in the absence as in the presence of the stimulus.

Geoffrey Hall concentrates on S-S learning: he aims to show that certain perceptual phenomena can be explained in terms of the associations used in animal-learning theory, a takeover bid that might have brought upon his head the resources of the Securities Council had he made it in the City (of London). His main target is a form of "perceptual learning" in which, under certain circumstances, organisms can learn to distinguish two stimuli to which they initially give identical responses; as a preliminary, he deals with a variety of other phenomena, including latent inhibition, the process by which repeated exposure to a stimulus reduces the stimulus's capacity to form associations.

Hall's arguments are based on many experiments, and are so detailed, subtle introduction to its true intellectual heirs - today's fast-growing neurosciences and linked developments in the computer simulation of neural processes. The heritage owed to the cybernetics movement could have been Heims's focusing theme. It may perhaps provide the theme of an inside story still to be written.

Donald Michie is at the Turing Institute, 36 North Hanover Street, Glasgow G1 2AD, UK.

and variegated that they cannot be summarized here. As a simple example of his theorizing, consider his account of "perceptual differentiation". A subject exposed to two stimuli subsequently shows an improved capacity to differentiate between them - for example, to learn one response to one, and a different response to the other. Hall's account depends on several assumptions, among them being that a stimulus is made up of "stimulus elements", that when an organism is exposed to a stimulus, salient elements acquire more latent inhibition than less salient ones, and that ability to discriminate between two stimuli is greater the higher the proportion of all stimulus elements not shared by the two stimuli. Hall postulates that each stimulus initially contains three different kinds of stimulus element: set 1 , those not shared with the other; set 2 , stimulus elements common to both stimuli; set 3 , novel elements, which Hall assumes are also common to both stimuli. Because the novel elements are, according to Hall, the most salient, the latent inhibition generated by exposure will reduce their power to form associations more than it reduces that of the stimuli in sets 1 and 2 . By reducing the novel elements, which are shared by both stimuli, preexposure will increase the proportion of stimulus elements that are specific to one or other stimulus. Hence, Hall claims, organisms will now discriminate between them more easily. Because novelty is not a property of the physical stimulus and must be detected by some such process as failing to match some elements of the stimulus to a stored representation, it seems odd to talk of "novel stimulus elements". Perhaps this does not affect Hall's argument.

Oddly, he does not extend his analysis to the phenomenon that many workers in visual perception regard as the most interesting example of perceptual learning. If someone views a range of similar objects such as cathedrals, he may at first find it hard to distinguish them, but with sufficient exposure he learns not only to distinguish those he has seen, but can on encountering a new example learn enough about it to tell it apart from all the others after a comparatively brief inspection. Hall could argue that all the "common elements" of cathedrals have been reduced in associative power by the previous exposures, making the features unique to particular examples more salient, so that discrimination becomes easier. But if the person is made to give a verbal response to common features, for example to say "flying buttress" or "triforium", there should be no latent inhibition and these features will remain salient: the differentiating features will then have no advantage and perceptual learning should not occur, an outcome that seems unlikely, particularly as attaching names to the parts seems to help recognition of the whole. The standard account of this type of learning given by workers on perception is that it is at first difficult to form a detailed representation of a structure as complex as a cathedral because there is too much new information. Once the appropriate representations are formed, however, much of the perceptual input can be mapped onto them. To construct an adequate representation of a new cathedral, all that is necessary is to modify the existing general representation in the few ways needed to specify that cathedral uniquely.

Such is Hall's ingenuity that he might well find a way of twisting this account into the language of latent inhibition, common stimulus elements and so on. But if animal learning is to take over perception, it must face such problems as which stimulus elements are common and which are not. Indeed, the use of the vague and entirely abstract expression "stimulus element" may be regarded as a retreat to mental atomism as put forward by John Locke. Animal-learning theorists take for granted the processing of both stimulus and response, and in thinking of associations, they make no use of the concept of hierarchy, without which no account of perception or indeed of any other aspect of cognition can be given. It may be partly for this reason that theories of animal learning, despite their liberation from the S-R approach, still seem rather impoverished.

Despite these qualms, Hall's book is a tour de force: it convincingly explains a wide range of phenomena, even if the nearer it gets to perception the less convincing it becomes. Hall is totally honest in reviewing both evidence and theories: he does not attempt to disguise inconsistencies in the former nor ambiguities in the latter. He suggests enough new experiments to keep workers in animal learning busy for years. And he exhibits great zest: one might say that he writes with faith, hope and clarity.

Stuart Sutherland is in the Department of Experimental Psychology, University of Sussex, Sussex House, Falmer, Brighton BN1 9QG, UK.

NATURE · VOL 356 · 12 MARCH 1992 